\title{
Phosphoglyceride crystal deposition disease originating from the myocardium
}

\author{
Fumihiro Shoji, MD, ${ }^{a}$ Ichiro Yoshino, MD, ${ }^{a}$ Takuro Kometani, MD, ${ }^{a}$ Hidetaka Yamamoto, MD,
} and Yoshihiko Maehara, MD, ${ }^{a}$ Fukuoka, Japan

rystal deposition diseases in the soft tissue are extremely rare and are encountered mostly in and around the joints, accompanied by arthritis. Several materials are known to be deposited as crystals, such as monosodium urate, calcium pyrophosphate, basic calcium phosphate (hydroxyapatite), and calcium oxalate. ${ }^{1,2}$ Phosphoglyceride crystal deposition disease (PCDD) is characterized by the deposition of crystals of phosphoglyceride, a phospholipid, in the injured tissues, although not in the joints. ${ }^{3}$ We herein report, for the first time, a patient presenting with an anterior mediastinal mass that was diagnosed by means of a surgical biopsy as a deposition of phosphoglyceride originating from the myocardial wound created at the time of surgical repair of a ventricular septal defect 35 years previously.

\section{Clinical Summary}

A 37-year-old man without any symptoms presented with an anterior mediastinal mass that was suspected of being a cardiac tumor or a thymic tumor invading the heart. Thirty-five years earlier, the patient underwent surgical repair for a ventricular septal defect. The laboratory data revealed no abnormality of the serum electrolytes, calcium level, phosphorus level, or blood count or the hepatic or renal function test results. Chest computed tomographic (Figure 1, A) and magnetic resonance imaging findings showed that an irregularly shaped and heterogeneously enhanced mass with partial calcification measuring $6 \mathrm{~cm}$ in size was located in the anterior mediastinum and extended into the myocardium. Ultrasonographic findings showed that the tumor most likely adhered to the anterior wall of the right ventricle (RV). In a fluorodeoxyglucose (FDG) positron emission tomographic scan (Figure $1, B)$, the mass showed high uptake of the FDG at a maximal standardized uptake value of 16.9. On the basis of these findings, a mediastinal tumor invading the RV or a cardiac tumor originating from the myocardium was suspected. A video-assisted thoracoscopic biopsy was performed to definitively establish the diagnosis. After dissecting the thickened pericardium, an elastic,

From the Departments of Surgery and Science ${ }^{\mathrm{a}}$ and Pathology, ${ }^{\mathrm{b}}$ Graduate School of Medical Sciences, Kyushu University, Fukuoka, Japan.

Received for publication April 9, 2007; accepted for publication April 20, 2007.

Address for reprints: Fumihiro Shoji, MD, Department of Surgery and Science, Graduate School of Medical Sciences, Kyushu University, 3-1-1, Maidashi, Higashi-ku, Fukuoka, 812-8582, Japan (E-mail: fshoji@ surg2.med.kyushu-u.ac.jp).

J Thorac Cardiovasc Surg 2007;134:508-9

$0022-5223 / \$ 32.00$

Copyright @ 2007 by The American Association for Thoracic Surgery doi:10.1016/j.jtcvs.2007.04.007 yellow-whitish-colored hard tumor was found at the anterior wall of the RV, and it was firmly fixed to the myocardium. An incisional biopsy was performed. Pathologic examinations revealed the following: (1) many eosinophilic crystals surrounded by histiocytes and multinucleated giant cells (Figure 2,A), the deposited material, which consisted of string-like crystals when viewed
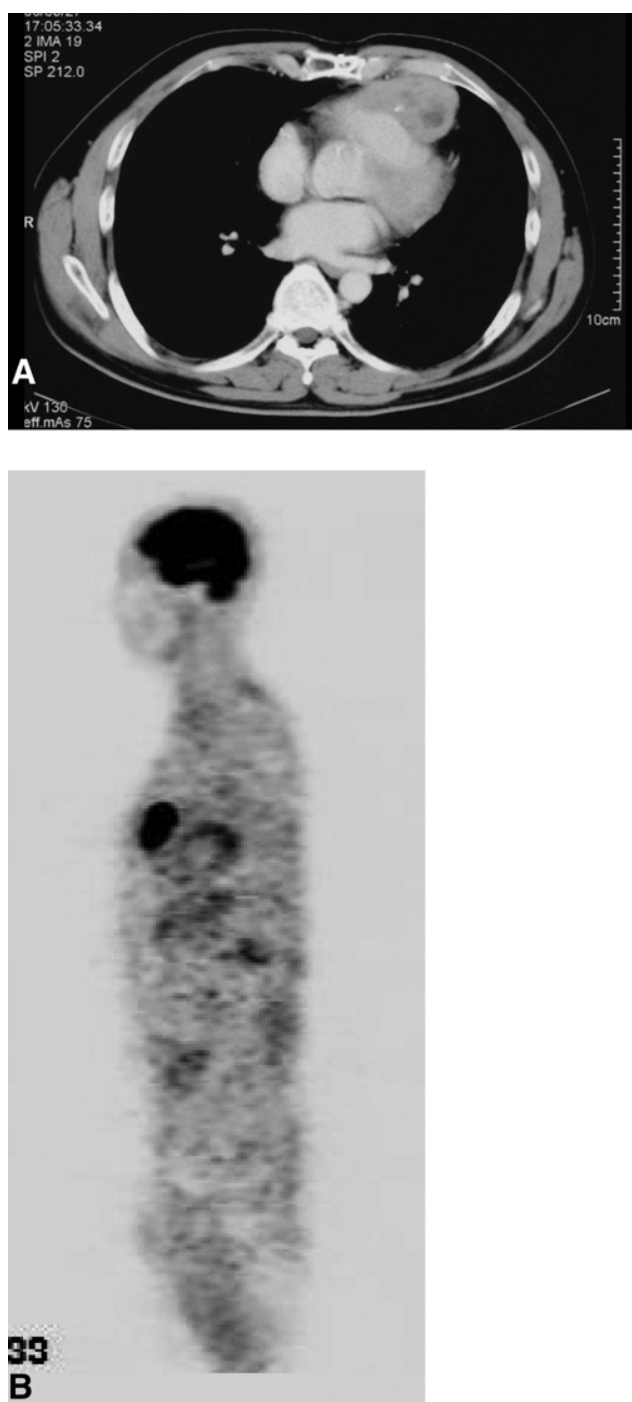

Figure 1. A, Chest computed tomographic scan showing a large and heterogeneous mass measuring $6 \mathrm{~cm}$ in size in the myocardium. B, Fluorodeoxyglucose positron emission tomographic scan showing a high uptake in the mass. 

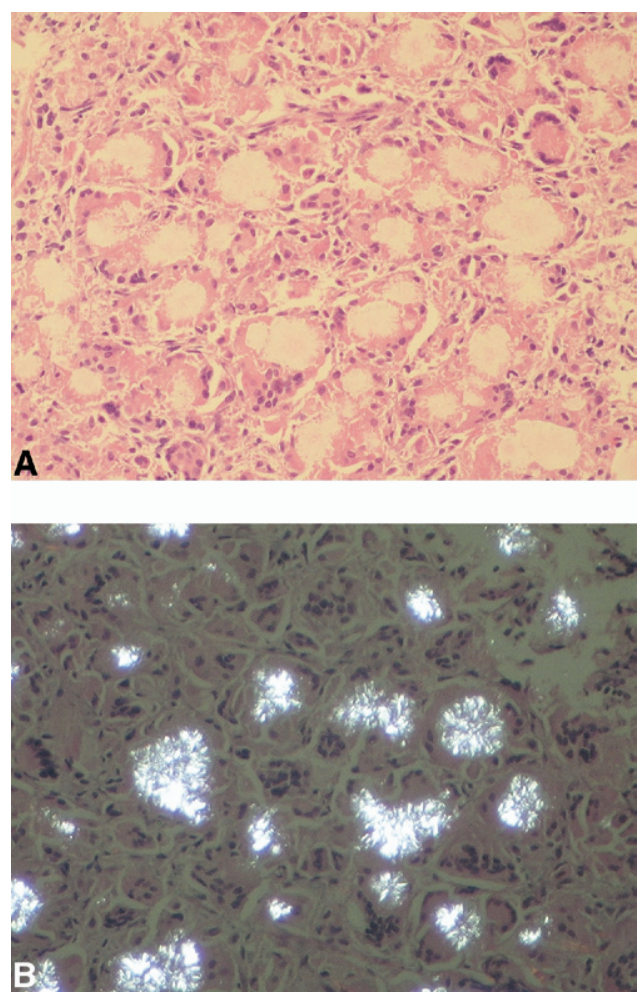

Figure 2. Histologic findings of the tumor. Many tiny granulomas with central bluish-pink crystals surrounded by macrophages were found. (Hematoxylin and eosin staining, original magnification $200 \times$.) The deposited material showed string-like crystals under polarized light, in which it appeared to be refractive.

under polarized light (Figure 2, B), and (2) immunohistochemically histiocytes and multinucleated giant cells, which were positive for vimentin and KP-1 (CD68). On the basis of these findings, the tumor was definitely diagnosed as a PCDD. The patient had an uneventful postoperative recovery and was discharged from the hospital, and he remains clinically stable 6 months after the operation.

\section{Discussion}

PCDD is a rare disease in which phosphohglyceride crystals are deposited, simulating a neoplasm in a scar of the soft tissue or bone. $^{1-5}$ In this case a myocardial scar where the PCDD was located had probably been made susceptible to this kind of disease. However, to the best of our knowledge, this is the first case arising from the internal organs.

Miura and colleagues ${ }^{4}$ summarized the clinical data of 6 patients with PCDD. The age of the patients ranged from 51 to 64 years, and there was no sex predilection, congenital abnormalities, metabolic disorders, or familial history of such diseases. The sites of deposition were characteristically intramuscular injection sites or postoperative sites, such as buttock muscle, brachial muscle, spine, gingival soft tissue, and skin incisions of the abdominal wall. ${ }^{1-5}$

The histologic findings of the PCDD are characterized by the aggregation of macrophages around the crystals and the formation of foreign body granulomas. On polarized microscopy, the fibrillar crystals appear refractive. The immunohistochemistry reveals macrophages accumulating around the many crystal deposits, which showed weak focal positivity for panmacrophage marker (CD68) and faint positivity for lysozyme and S-100 protein. ${ }^{4}$

The PCDD was reported to have gradually increased in size over more than 10 years and to recur repeatedly after surgical removal. Therefore, the tendency is to diagnose the PCDD as a neoplasm. ${ }^{4} \mathrm{We}$ also suspected that the lesion in this case was a malignant tumor from the findings of the computed tomographic scan and the fluorodeoxyglucose positron emission tomographic scan at the time of the initial presentation. In most PCDDs excisional surgery should be performed, if possible. However, in this case complete resection was not possible because of the firm adhesion of the lesion to the RV. Thus we must continue to follow-up this patient carefully. In conclusion, this rare case revealed that the PCDD could occur even in a surgical scar in the heart.

\section{References}

1. Miura K, Fukuda H, Mineta H, et al. Phosphoglyceride crystal deposition disease. Pathol Int. 2000;50:992-8.

2. Kubo K, Manabe J, Matsumoto S, et al. Unusual huge intramuscular granuloma with calcium phosphate crystal deposition in the buttock. Acta Pathol Jpn. 1992;42:508-11.

3. Yachida S, Fukushima N, Nakanishi Y, et al. Phosphoglyceride crystal deposition disease. Presenting as multiple paragastric masses. Dig Dis Sci. 2002;47:1413-8.

4. Miura K, Han G, Kondo S, et al. Phosphoglyceride (PG) crystal deposition disease: a novel acquired disease in which PG crystals are deposited in injured soft tissue and bone with phospholipid metabolism disturbance. Pathol Int. 2004;54:881-9.

5. Nishiumura A, Kasai Y, Takegami K, et al. Phosphoglyceride crystal deposition disease in the spine: a case report. J Spinal Disord Tech. 2005;18:112-5. 